Revue des patrimoines

$44 \mid 2021$

Les patrimoines des lycées français du XIXe au XXIe siècle : de la connaissance à la valorisation

\title{
Les patrimoines des lycées français
}

$\mathrm{XIX}^{\mathrm{e}}-\mathrm{XXI}^{\mathrm{e}}$ siècles

Cécile Fabris, Catherine Guégan, Éric Cron et Jean Davoigneau

\section{(2) OpenEdition}

\section{Journals}

Édition électronique

URL : https://journals.openedition.org/insitu/30829

DOI : 10.4000/insitu.30829

ISSN : 1630-7305

Éditeur

Ministère de la Culture

Référence électronique

Cécile Fabris, Catherine Guégan, Éric Cron et Jean Davoigneau, « Les patrimoines des lycées

français », In Situ [En ligne], 44 | 2021, mis en ligne le 30 avril 2021, consulté le 16 décembre 2022.

URL : http://journals.openedition.org/insitu/30829; DOI : https://doi.org/10.4000/insitu.30829

Ce document a été généré automatiquement le 16 décembre 2022.

\section{c) (i) $\Theta$}

Creative Commons - Attribution - Pas d'Utilisation Commerciale - Pas de Modification 4.0 International - CC BY-NC-ND 4.0

https://creativecommons.org/licenses/by-nc-nd/4.0/ 


\title{
Les patrimoines des lycées français
}

$\mathrm{XIX}^{\mathrm{e}}-\mathrm{XXI}^{\mathrm{e}}$ siècles

\author{
Cécile Fabris, Catherine Guégan, Éric Cron et Jean Davoigneau
}

1 L'intérêt pour les patrimoines des lycées français reste relativement récent dans l'historiographie des études sur l'architecture scolaire. Les historiens de l'éducation avaient bien naturellement ouvert la voie à ces recherches, tel Paul Gerbod dont l'ouvrage précurseur reste encore une référence ${ }^{1}$. Sans chercher à être exhaustif, tant les domaines sont vastes - du cadre législatif et règlementaire à la définition des programmes et de leurs supports en passant par la formation des enseignants, la vie scolaire ou les bâtiments et le mobilier-, il est important de citer ici le colloque organisé en 2002 par le centre Roland-Mousnier de l'université Paris IV-Sorbonne et l'Institut national de recherche pédagogique (INRP) à l'occasion du bicentenaire de la loi du $1^{\mathrm{er}}$ mai 1802 instituant les lycées. Publié en 2005, il constitue une excellente entrée en matière et présente à la fois des synthèses sur certains sujets et de nouvelles perspectives de recherche ${ }^{2}$. Dans le même ordre d'idées, il convient de citer les travaux d'Antoine Prost ${ }^{3}$, de Philippe Marchand ${ }^{4}$, de Philippe Savoie ${ }^{5}$ et de Solenn Huitric ${ }^{6}$, indispensables à l'historien de l'architecture pour étudier le développement du réseau des lycées en France. Si l'enseignement général est à l'honneur dans ces publications, des approches thématiques - il est vrai plus rares - offrent également de précieux éléments de contexte pour comprendre la diversification et la spécialisation de certains établissements, en particulier en matière d'instruction technique ${ }^{7}$ et agricole ${ }^{8}$.

2 Si l'on excepte les premières histoires architecturales des collèges de l'Ancien Régime, dans lesquels certains établissements de la première génération se sont installés, les études sur l'architecture des lycées sont donc plus récentes et même en décalage par rapport à celles sur les écoles primaires. Ce constat est d'autant plus étonnant que la littérature et les revues spécialisées des $\mathrm{XIX}^{\mathrm{e}}$ et $\mathrm{XX}^{\mathrm{e}}$ siècles n'ont pas omis de traiter le sujet. L'article «Lycée» publié par l'architecte Charles Lucas (1838-1905) dans l'Encyclopédie de l'architecture et de la construction, éditée entre 1888 et 1892 sous la direction de Paul Planat, y consacra ainsi de nombreuses pages. L'Architecture d'aujourd'hui publia en 1951 un numéro spécial sur les constructions scolaires et l'année suivante, ce fut au tour de L'Architecture française de faire de même, à la demande du 
ministère de l'Éducation nationale, en écho à l'exposition organisée à l'École nationale des beaux-arts. Le même constat s'applique enfin à une autre revue spécialisée, Technique et architecture, qui traita plus particulièrement le sujet à quatre reprises en 1955, 1956, 1957 et 1960. Il convient de préciser que cette fréquence des publications est liée bien évidemment au contexte de l'après-guerre et à la vaste entreprise d'industrialisation de la construction scolaire alors à l'œuvre.

3 Les études sur l'architecture et les objets mobiliers des lycées se sont néanmoins multipliées ces dernières années. Hormis quelques approches strictement monographiques plus anciennes, les recherches et les publications de Marc Le Cœur, auteur d'une thèse sur les lycées parisiens, restent parmi les premières du genre et ont eu de surcroît l'ambition de présenter rapidement des synthèses nationales sur plusieurs thèmes'. Une entreprise comparable est à mettre à l'actif de Francis Gires qui s'est spécialisé dans l'étude des objets scientifiques et techniques de l'enseignement. Ses publications n'ont pas seulement une valeur scientifique mais également une portée méthodologique et sont aujourd'hui de véritables outils de travail pour les chercheurs $^{10}$. Depuis la loi de 2004 relative aux libertés et responsabilités locales, qui a décentralisé la compétence de l'Inventaire général du patrimoine culturel au profit des régions, les patrimoines des lycées sont devenus en outre des thèmes privilégiés des programmations scientifiques de ces services - une dizaine les ayant étudiés ou étant en train de le faire. Cette dynamique de recherche, exceptionnelle à bien des égards, est donc en passe aujourd'hui de renouveler en profondeur la connaissance sur la construction et le fonctionnement des lycées en France.

4 Si le temps de la synthèse nationale pour les services régionaux de l'Inventaire n'est pas encore arrivé, plusieurs publications monographiques grand public ont vu le jour dans ce cadre ${ }^{11}$. Un groupe national sur l'étude des lycées a par ailleurs été constitué et a organisé cinq séminaires professionnels sur le sujet en collaboration avec la mission Inventaire général du patrimoine culturel du ministère de la Culture, les Archives nationales et le musée national de l'Éducation à Rouen. Ces séminaires ont permis aux chercheurs des différents services régionaux de confronter sources dépouillées, approches méthodologiques, thèmes d'études spécifiques. Une dizaine d'articles des deux numéros de la revue In Situ consacrés aux patrimoines des lycées, que nous présentons ici, présentent d'ailleurs un large panorama de ces travaux déjà réalisés ou toujours en cours. De même, trois autres articles publiés dans des numéros thématiques antérieurs de la revue In Situ témoignent de la diversité des types d'enquêtes menées par les services régionaux de l'Inventaire: enseignement professionnel ${ }^{12}$, objets et mobiliers pédagogiques ${ }^{13}$, œuvres du $1 \%$ artistique $^{14}$. Quelques équipes commencent par ailleurs à restituer leurs travaux menés à l'échelle régionale. Dès 2013, le service Inventaire et Patrimoine de la Région Franche-Comté a organisé un colloque intitulé «Enseignement technique et Architecture » afin de présenter un premier bilan des études menées sur les lycées professionnels ${ }^{15}$. Des journées d'études consacrées aux patrimoines des lycées ont été organisées par la Région Nouvelle-Aquitaine les 10 et 11 octobre 2019 au lycée Grand Air d'Arcachon, qui ont permis d'esquisser quelques éléments de synthèse ${ }^{16}$. Un colloque s'est tenu les mêmes jours à l'université de Bourgogne sur "Ce que l'art fait à l'école. La politique publique d'insertion d'œuvres plastiques dans les établissements scolaires et universitaires. 1880-2020». Enfin, en Auvergne-Rhône-Alpes, un premier article mis en ligne en 2020 a posé les bases d'une analyse sur la place dulycée dans la ville ${ }^{17}$. Ces initiatives sont d'autant plus importantes que la connaissance et la sensibilisation progressive qui en découlent se 
mettent progressivement au service des politiques publiques en faveur de la valorisation du patrimoine.

Or, sur ce dernier point, beaucoup de choses restent à faire, à commencer par la sensibilisation des maîtres d'ouvrage, qui s'avère d'autant plus indispensable que les plans prévisionnels d'investissement sur les lycées sont généralement bien dotés dans chaque région. La prise en compte de la dimension patrimoniale dans les projets de réhabilitation constitue donc un enjeu à part entière, ce qui ne va pas forcément de soi pour l'ensemble du corpus et notamment pour les édifices de la seconde moitié $\mathrm{du} \mathrm{Xx}{ }^{\mathrm{e}}$ siècle. Mais le terrain à conquérir concerne également en premier lieu les services patrimoniaux eux-mêmes. Sur 45832 édifices protégés au titre des monuments historiques $^{18}$, on compte seulement 96 lycées, soit environ $0,2 \%$ du corpus. Seul un tiers d'entre eux bénéficie par ailleurs d'une mesure de classement total ou partiel. Parmi cette centaine d'édifices scolaires : 72 lycées véritables, 13 anciens collèges d'Ancien Régime devenus lycées; 13 construits au XIX siècle et 17 au XX ${ }^{\mathrm{e}}$ siècle (dont 12 de la première moitié et 5 de la seconde). Le plus contemporain protégé, le lycée agricole François Pétrarque à Avignon (Vaucluse) construit de 1966 à 1969 par les architectes Roland Bechmann, Pierre Biscop, Charles André et François Girard a été inscrit au titre des monuments historiques dès novembre 1989. En ce qui concerne le $\mathrm{XX}^{\mathrm{e}}$ siècle, le lycée, comme type architectural, s'avère être un peu mieux pris en compte par le label architecture contemporaine remarquable, il représente près de $4 \%$ du corpus (61 édifices sur 1583 labellisés ${ }^{19}$ ), mais ce pourcentage retombe à $1,5 \%$, si l'on retire du total des labellisés les 38 lycées d'Île-de-France qui viennent d'obtenir le label en 2020 et 2021 à l'issue d'une opération singulière menée conjointement par le service d'Inventaire de la Région et la DRAC. La faible représentation des lycées labélisés "Architecture contemporaine remarquable» ou protégés au titre des monuments historiques démontre, s'il le fallait, la nécessité de faire évoluer sans cesse les contours de ce que nous appelons habituellement patrimoine. Au même titre que la recherche proprement dite, une évidence s'impose à nous en ce qui concerne les lycées : par son histoire et l'importance du cadre législatif et règlementaire, le périmètre de réflexion ne peut être que national.

\section{Un cadre de réflexion national}

6 La Constitution de 1791 affirme dans ses Dispositions fondamentales le caractère public de l'instruction: «Il sera créé et organisé une Instruction publique commune à tous les citoyens, gratuite à l'égard des parties d'enseignement indispensables pour tous les hommes et dont les établissements seront distribués graduellement, dans un rapport combiné avec la division du royaume. »Ce principe fondateur a été retranscrit dans la loi du 11 floréal an X ( $1^{\mathrm{er}}$ mai 1802) qui institue trois types d'établissements : les écoles primaires, les écoles secondaires établies par des communes ou des particuliers et les lycées financés par l'État. Si la première pierre du système scolaire public venait d'être posée, l'idée qu'il convenait de se doter d'édifices dédiés à l'enseignement faisait également son chemin. Tout restait néanmoins à écrire, que ce soit pour les programmes ou pour les prescriptions architecturales destinées à définir, organiser et hiérarchiser les bâtiments. Ces deux axes n'évoluèrent pas forcément de manière strictement parallèle, mais restèrent néanmoins liés et furent constamment réinterrogés tout au long des $\mathrm{XIX}^{\mathrm{e}}$ et $\mathrm{XX}^{\mathrm{e}}$ siècles. 
7 Chaque époque apporta ainsi sa pierre à l'édifice. Avec la création des lycées en 1802, le Premier consul avait voulu renouer avec la tradition des collèges d'humanités qui avaient été supprimés par la Révolution. L'enjeu éminemment politique visait à restaurer la puissance de l'État grâce à la formation des élites masculines. Afin de prendre en charge au plus tôt ces futurs cadres, les lycées furent même dotés de classes élémentaires et primaires. Ils n'avaient toutefois pas le monopole de l'enseignement secondaire, puisque la création d'écoles communales était également prévue. Deux cycles parallèles, et non successifs, coexistèrent donc et furent régis par des critères sociaux et culturels, entretenant par là-même une logique de classe. Dès les premières années, la direction de l'Instruction publique se heurta à des difficultés liées en premier lieu à une répartition géographique inégale des établissements et au recrutement du corps enseignant. Elle n'en fut pas moins ambitieuse avec l'objectif de porter le nombre de lycées à plus de cent (décret du 15 novembre 1811) - objectif qui ne fut toutefois atteint qu'en 1887.

Alors que les premiers lycées s'étaient installés dans des bâtiments existants, le développement du réseau au $\mathrm{XIX}^{\mathrm{e}}$ siècle, en particulier pendant la monarchie de Juillet et les années 1840, obligea l'administration de l'Instruction publique à mieux encadrer ces créations et finalement les premières constructions. Elle rédigea plusieurs règlements - en 1802,1843, 1861, 1881, 1891 et 1929 - qui, empiriques dans un premier temps, se nourrirent ensuite des avancées dans les champs de la science, de la pédagogie et de l'hygiène pour devenir de plus en plus normatifs et mieux répondre aux enjeux de fonctionnalité, de prophylaxie et de moralité. Cette naissance progressive d'une architecture publique dédiée et les inévitables débats sur la conception d'un lycée participèrent par ailleurs aux controverses qui agitaient alors l'École des beaux-arts et l'Académie d'architecture, ainsi que les tenants d'une nouvelle pensée plus sensible au rationalisme structurel. Sous la Troisième République, le réseau poursuivit son développement dans un contexte de laïcisation de l'éducation. Afin de mieux être en phase avec son temps, une vaste entreprise de démocratisation et de diversification de l'enseignement secondaire fut engagée, en particulier en direction des filles (loi du 21 décembre 1880 du député Camille Sée) et des filières techniques et agricoles.

Dès la fin de la Première Guerre mondiale, des critiques condamnèrent la double voie créée en 1802. Des mesures législatives décisives tentèrent alors d'atténuer la logique de classe induite par ce système : la gratuité du cycle secondaire des lycées fut obtenue en 1930 et l'obligation scolaire fut prolongée de 13 à 14 ans par Jean Zay. Si la création en 1963 des collèges d'enseignement secondaire priva progressivement les lycées des classes du premier cycle, l'unification ne s'acheva toutefois qu'avec la loi Haby du 11 juillet 1975 qui instaura un collège unique pour tous entre l'école primaire et le lycée. Parallèlement à ces évolutions, la France dut faire face à une augmentation significative des effectifs, qui passèrent de 326000 lycéens à la fin des années 1940 à plus d'un million au début des années 1960. La croissance démographique, le besoin d'une main d'œuvre plus instruite et la prolongation de la scolarité obligatoire jusqu'à 16 ans en 1959 furent les causes premières d'une telle expansion. Dans ce contexte, l'État et en particulier la direction des Équipements scolaires, universitaires et sportifs créée en 1956 au sein du ministère de l'Éducation nationale ne pouvaient que reprendre la main sur les questions de construction et de règlementation avec l'objectif d'industrialiser le processus de construction. 
Inadaptées aux évolutions de la pédagogie, les constructions dites «industrielles » furent toutefois contestées dès le début des années 1970. Elles furent même jugées dangereuses après l'incendie tristement célèbre du collège de la rue Pailleron à Paris, qui fit vingt victimes le 6 février 1973. Les lois de décentralisation des 7 janvier et 22 juillet 1983 (effectives au $1^{\mathrm{er}}$ janvier 1986), confiant aux régions la gestion des lycées, ont ouvert alors une nouvelle phase de l'histoire de ces établissements scolaires. Outre la nécessaire réhabilitation et mise aux normes des bâtiments, les programmes s'enrichissent graduellement pour faire des lycées des lieux de vie : amphithéâtre, salle polyvalente, CDI (centre de documentation et d'information), cafétéria, bureaux pour recevoir les parents d'élèves, etc. Ces réflexions se poursuivent aujourd'hui autour des enjeux de la consommation d'énergie et de l'accessibilité mais également de la place du numérique dans les enseignements, tentant d'inventer ce que certains appellent le lycée du futur. Dans ce contexte, la prise de conscience de l'importance de l'histoire au long cours des lycées et de la qualité patrimoniale des établissements reste un enjeu de premier ordre. Il ne s'agit pas seulement d'étudier pour comprendre et participer à l'élaboration d'un récit national mais de sensibiliser l'ensemble des acteurs pour que les différentes composantes d'un lycée - l'architecture, les compositions paysagères, le mobilier, les collections pédagogiques, le $1 \%$ artistique - soient prises en compte dans l'adaptation et la modernisation de ces lieux d'éducation. Ce numéro de la revue In Situ ainsi que celui qui suit ont l'ambition d'y contribuer.

\section{Les problématiques abordées dans les deux numéros}

11 L'étude du patrimoine des lycées recouvre des aspects protéiformes, variant points de vue et perspectives, que les contributions ici proposées mettent en évidence. La diversité des champs explorés en témoigne: qu'il s'agisse des rapports entre collectivités maîtres d'ouvrage et administrations de l'État en termes de programmes, d'élaboration de projets et de réalisation (Solenn Huitric \& Julien Cahon), du rôle des architectes et maîtres d'œuvre dans la constitution d'un ensemble de normes et de modèles dès l'époque moderne ou de la mise en place précoce de formes de contrôle visant à organiser et normaliser la construction des établissements (Catherine Guégan). Anne-Marie Châtelet et Claude Laroche s'attachent ainsi à montrer combien, tout au long du XIXe siècle, l'érection des lycées se définit comme le champ d'enjeux aussi bien théoriques que programmatiques où s'affrontent et s'affirment différents courants et écoles de pensée, essentiellement au sein des structures académiques mais aussi en dehors d'elles.

L'exceptionnelle richesse de la documentation produite, sans laquelle la mise au jour de cet ensemble de processus ne pourrait se faire et, parfois, la complexité de son organisation, nécessitaient un indispensable éclairage de la part de ceux qui ont mission de collecter, conserver et valoriser les archives (Cécile Fabris). Leurs contributions participent par ailleurs pleinement de l'actualité de la recherche en matière d'histoire de l'enseignement secondaire et de ses établissements, qu'il s'agisse de la conception, de la construction et du fonctionnement des lycées durant les Trente Glorieuses (Anne Rohfritsch) ou de leur décoration (Pascal Riviale \& Clothilde Roullier), dont une récente exposition aux Archives nationales a exploré toutes les facettes ${ }^{20}$. Elles rappellent que le travail indispensable de collecte et de valorisation des sources, s'agissant du patrimoine des lycées, est mené à tous les niveaux - national, 
régional (Muriel Bessot \& Agnès Dejob) ou départemental (Isabelle Girard) - en lien soit avec une institution (Franck Delorme) soit avec un établissement (Maylis Sanchis, Romain Joulia \& Liliane Hamelin). Ce patrimoine touche en outre à l'immatériel dès lors que l'on s'intéresse aux pratiques pédagogiques contemporaines et aux nouveaux usages en milieu scolaire (éducation à la citoyenneté, conseils de vie lycéenne, sensibilisation aux discriminations), ce qui nécessite d'innover dans l'entreprise de collecte, ainsi que le montre l'article de Nicolas Coutant au moyen de trois exemples marquant cette entrée de nouveaux « objets » dans les collections du musée national de l'Éducation (Rouen). Enfin, au-delà de leur statut de sources, les ensembles archivistiques participent également du patrimoine des lycées, objet de pratiques de valorisation particulières (Isabelle Girard, olga Yardin \& Sébastien Chevereau).

13 La question de savoir comment le lycée se constitue en tant qu'objet patrimonial est au cœur des études de cas regroupées dans les deux numéros. Couvrant une large étendue du territoire français, y compris ultramarin, les contributions de Clémence Préault sur le lycée de Saint-Denis de La Réunion, de Thierry Goyet sur les lycées de la Reconstruction à Lorient (Morbihan) ou de Robert Blaizeau sur celle du lycée Le Verrier à Saint-Lô (Manche), de Bertrand Charneau sur celui de Talence (Gironde), d'Élise Nale \& Martine Tronquart sur le lycée Paul-Louis Cifflé à Nancy (Meurthe-et-Moselle), de Karine Girard sur la cité scolaire Eugène-Thomas du Quesnoy (Nord), de Pierre Maurer sur les lycées de Metz (Moselle), confrontent dans la diachronie ou la synchronie les modalités qui ont donné lieu à la création de ces établissements, leur évolution au gré des politiques pédagogiques et constructives de l'État, et leur progressive affirmation dans le champ patrimonial. Elles permettent de cerner la mise en œuvre de ces politiques, notamment durant la période des Trente Glorieuses, et leur nécessaire adaptation aux contextes locaux et aux besoins des territoires en matière d'enseignement professionnel. Le transfert des lycées aux régions en 1986 marque probablement un tournant, autorisant plus de variété dans les implantations, les formes, les matériaux (Pierre Maurer), même si l'État continue à élaborer les règles et les normes en matière de pédagogie et d'organisation des locaux. Enfin, en ciblant les installations sportives des lycées, lesquelles ont peu retenu l'attention jusqu'à présent, Philippe Bonnet élargit le champ patrimonial de ces derniers et administre la preuve de l'intérêt que leur étude revêt, dans la mesure où ils révèlent, eux aussi, les vicissitudes des politiques étatiques d'une institution qui fut l'un des principaux vecteurs du développement des pratiques sportives en France. Il en va de même s'agissant de l'utilisation de matériaux qui inscrivent de nouvelles pratiques constructives issues du progrès des techniques dans le champ de la création au service d'«espaces d'hospitalité » (Yacine Kébir).

14 Le champ patrimonial ne recouvre donc pas les seuls édifices mais tout un ensemble d'éléments structurels et d'objets, qu'ils relèvent de l'histoire de l'enseignement et des pratiques pédagogiques (c'est le cas des instruments scientifiques et techniques, des spécimens animaux ou minéraux et de bien d'autres encore) ou artistiques (les collections de modèles antiques en plâtre, éléments essentiels de la pédagogie artistique au XIX ${ }^{\mathrm{e}}$ siècle). Cet ensemble hétérogène constitue aujourd'hui le fonds de collections d'une valeur parfois inestimable, peu connues du grand public, dont Henri Chamoux \& Françoise Khantine-Langlois mettent en évidence tant l'intérêt que la fragilité, au vu des périls que constituent l'oubli, la méconnaissance, ou la nécessité de «faire de la place», et les différentes modalités d'action mises en œuvre pour étudier, porter à connaissance et valoriser ce "patrimoine caché »: inventaires en 
ligne, publications, création d'espaces "muséaux» au sein des établissements, expositions virtuelles. Celle des collections du lycée Émile-Zola de Rennes (Ille-etVilaine), portée par la Région Bretagne via son service de l'Inventaire du patrimoine et ici présentée par Justine Malpeli, est le fruit d'une collaboration inédite avec l'Amélycor (Association pour la mémoire du lycée et du collège de Rennes) et d'un partenariat technologique avec Google, qui, sur sa plateforme Google Arts \& Culture, permet de découvrir à distance un " cabinet de curiosité " pédagogique.

De ce patrimoine relèvent également les œuvres commandées depuis 1951 par l'État et les collectivités pour orner leurs établissements scolaires, les « $1 \%$ artistiques » dont Philippe Mairot \& Guillaume Capou, après en avoir dressé le tableau sur le territoire de la région Bourgogne-Franche-Comté, soulignent combien il peut être lui aussi menacé et - ou parce que - méconnu de ceux qui le côtoient quotidiennement, souvent, mais aussi comment médiation et valorisation peuvent contribuer à le réintégrer au cœur de la cité scolaire. C'est également dans cette optique qu'intervient au lycée Émile-Zola de Rennes la danseuse et chorégraphe Léna Massiani, proposant une approche du patrimoine par la danse, dont la présente contribution expose le projet. La Région Nouvelle-Aquitaine a fait pour sa part le pari ambitieux, et réussi, d'associer les lycéens dans une démarche participative à son opération d'inventaire du patrimoine des lycées à travers un dispositif d'éducation artistique et culturelle, " Histoire de bahuts », visant à les sensibiliser à l'histoire et à l'architecture de leur établissement (Laetitia Maison). Ainsi s'ouvrent de nouvelles perspectives de recherche et de valorisation des patrimoines des lycées. Ces actions de valorisation et de protection du patrimoine au sein des établissements influent-elles sur l'évolution des principes et des pratiques des usagers des lycées? C'est la question que posent Sylvain Wagnon \& Fabien Groeninger.

16 L'un des objectifs des deux numéros d'In Situ dédiés aux lycées est bien de s'attacher à décrire les processus de patrimonialisation à l'œuvre et de leur donner une visibilité nouvelle. C'est à cette dernière tâche que se sont attelés certains services patrimoniaux de l'État et des régions, dont les efforts en matière de protection méritent d'être soulignés, voire encouragés. Le service de l'Inventaire de la Région Île-de-France a ainsi conduit une opération inédite, conjointement avec la direction régionale des Affaires culturelles (Drac), visant à proposer une quarantaine d'entre eux au label «Architecture contemporaine remarquable» attribué par le ministère de la Culture afin de distinguer leur valeur historique, architecturale et artistique (Emmanuelle Philippe \& Marianne Mercier). Cependant, les efforts déployés n'aboutissent pas toujours aux résultats espérés (Michèle François), et des actions de sensibilisation restent à porter auprès des décideurs pour mettre en lumière l'intérêt de bâtiments souvent considérés comme des "usines à enseigner" et que de piètres qualités thermiques et acoustiques exposent à un risque de disparition sinon totale du moins partielle. Où se pose ici la question de ce qui est communément perçu comme faisant patrimoine - auquel une attention particulière est portée de longue date - et des moyens engagés pour le préserver et le mettre en valeur, parfois en l'affectant à de nouveaux usages, telle la chapelle du lycée Corneille de Rouen, en Seine-Maritime (Bénédicte Duthion). 


\section{NOTES}

1. GERBOD Paul, La Vie quotidienne dans les lycées et collèges au XIX siècle, Paris, Hachette, 1968.

2. CASPARD Pierre, LUC Jean-Noël \& SAVOIE Philippe (dir.), Lycées, lycéens, lycéennes. Deux siècles d'histoire, Paris, INRP, 2005.

3. PROST Antoine, «Les grandes étapes de l'histoire des lycées de 1802 à 1902 », in CHARMASSON Thérèse \& LE GOFF Armelle (dir.), Mémoires de lycées. Archives et patrimoine, actes de la journée d'études du 8 juillet 2002 organisée par le Centre historique des Archives nationales (Paris, hôtel de Rohan), Paris, direction des Archives de France / INRP, 2003.

4. MARCHAND Philippe, " Histoire et commémoration : le bicentenaire des lycées (1802-2002)», Histoire de l'éducation, $\mathrm{n}^{\circ} 109$, «Varia», 2006, p.75-117, [disponible en ligne] https:// journals.openedition.org/histoire-education/1330?lang=fr [lien valide en mars 2021].

5. SAVOIE Philippe, La Construction de l'enseignement secondaire (1802-1914). Aux origines d'un service public, Lyon, ENS éditions, coll. «Hors collection», 2013, disponible en ligne https:// books.openedition.org/enseditions/5044 [lien valide en mars 2021].

6. HUITRIC Solenn, «Transformer les collèges communaux en lycées. La coproduction d'une action publique (1830-1880)», thèse de doctorat d'histoire sous la direction de Philippe Savoie soutenue à l'École normale supérieure de Lyon / université de Lyon, 2016.

7. BELHOSTE Bruno, «L'Enseignement secondaire français et les sciences au début du $\mathrm{xx}^{\mathrm{e}}$ siècle. La réforme de 1902 des plans d'études et des programmes », Revue d'histoire des sciences, t. 43, $n^{\circ} 4$, "L'enseignement scientifique au tournant des XIX et $\mathrm{Xx}^{\mathrm{e}}$ siècles », 1990, p. 371-400, [disponible en ligne ] https://www.persee.fr/doc/rhs_0151-4105_1990_num_43_4_4502 [lien valide en mars 2021] ; LEMBRÉ Stéphane, "L'Histoire de l'enseignement technique et professionnel » in CONDETTE Jean-François \& FIGEAC-MONTHUS Marguerite (dir.), Surlestraces du passé de l'éducation... Patrimoines et territoires de la recherche en éducation dans l'espace français, Pessac, Maison des sciences de l'homme d'Aquitaine, coll. «Élites politiques et idéologie », 2014, p. 273-281.

8. CHARMASSON Thérèse, LELORRAIN Anne-Marie \& RIPA Yannick (dir.), L'Enseignement agricole et vétérinaire, de la Révolution à la Libération: textes officiels, Paris, INRP / Publications de la Sorbonne, 1992.

9. LE CCEUR Marc, «Les lycées dans la ville: l'exemple parisien (1802-1914)», Histoire de l'éducation, $\mathrm{n}^{\circ} 90$, «L'établissement scolaire. Des collèges d'humanités à l'enseignement secondaire, $\mathrm{XVI}^{\mathrm{e}}-\mathrm{Xx}^{\mathrm{e}}$ siècles ", 2001, [disponible en ligne] https://journals.openedition.org/ histoire-education/835 [lien valide en mars 2021] ; LE CđEUR Marc \& CHÂTELET Anne-Marie (dir.), Histoire de l'éducation, $\mathrm{n}^{\circ}$ 102, "L'Architecture scolaire. Essai d'historiographie internationale ", 2005 [2004], [disponible en ligne] https://journals.openedition.org/histoire-education/639 [lien valide en mars 2021]; LE CEUUR Marc, «Couvert, découvert, redécouvert... L'invention du gymnase scolaire en France (1818-1872) », Histoire de l'éducation, n 102, « L'Architecture scolaire. Essai d'historiographie internationale », 2005 [2004], p. 109-135, [disponible en ligne] https:// journals.openedition.org/histoire-education/705 [lien valide en mars 2021]; LE CEUR Marc, "L'architecture et l'installation matérielle des lycées: la règlementation et sa mise en œuvre (1802-1940) », in CASPARD Pierre, LUC Jean-Noël \& SAVOIE Philippe, Lycées, lycéens, lycéennes, p. 363-380 ; LE CEUUR Marc, «La chaire et les gradins. De la salle de classe à la salle de cours dans les lycées au XIX ${ }^{\mathrm{e}}$ siècle ", Histoire de l'éducation, $\mathrm{n}^{\circ} 130$, "Le cours magistral XIX $\mathrm{XX}^{\mathrm{e}}$ siècle. 2. Le cadre institutionnel et matériel », 2011, p.85-109, [disponible en ligne] https:// journals.openedition.org/histoire-education/2331 [lien valide en mars 2021]; LE CEUR Marc, «Un cas d'école : les lycées », Livraisons d'histoire de l'architecture, n 21, « Bâtir et orner », 2011, 
p. 89-100, [disponible en ligne] https://journals.openedition.org/lha/279 [lien valide en mars 2021]

10. GIRES Francis (dir.), Physique impériale. Cabinet de physique du lycée impérial de Périgueux, catalogue d'exposition (Pau, Bordeaux, Périgueux, Paris, Ivry, 2003-2007), Niort, Association de sauvegarde et d'étude des instruments scientifiques et techniques de l'enseignement (ASEISTE), 2005 ; GIRES Francis (dir.), L'Empire de la physique. Cabinet de physique du lycée Guez-de-Balzac d'Angoulême, catalogue réalisé à l'occasion du centenaire du Bulletin de l'Union des physiciens (1907-2007), en hommage à Ernest Fourteau (Périgueux, 1840-Paris, 1915), Niort, ASEISTE, 2006 ; GIRES Francis (dir.), L'Empire des sciences naturelles. Cabinets d'histoire naturelle des lycées impériaux de Périgueux et d'Angoulême, Niort, ASEISTE, 2013 ; GIRES Francis (dir.), Encyclopédie des instruments de l'enseignement de la physique du XVIII au milieu du XXe siècle, Niort, ASEISTE, 2016.

11. POUPARD Laurent, BERMOND Sandrine, CHARENTON Thomas, JACOUTOT François, RICHARD Joël \& SCHEID Maguy, L'École d'horlogerie de Besançon, le lycée Jules-Haag, Lyon, Lieux Dits, coll. « Parcours du patrimoine », 2012; POUPARD Laurent, et al., L'École d'optique de Morez, le lycée Victor-Bérard, Lyon, Lieux Dits, coll. «Parcours du patrimoine », 2013 ; DUTHION Bénédicte, Du collège des Jésuites au lycée Corneille, Rouen, Lyon / Rouen, Lieux Dits / Région HauteNormandie, coll. « Patrimoine et territoire », 2015 ; GIRARD Karine \& RÉGION HAUTS DE FRANCE, Le Lycée Gambetta de Tourcoing. Une histoire militante, Lyon, Lieux Dits, coll. «Images du patrimoine ", 2017 ; GUÉGAN Catherine, Lycée Ampère, Lyon. Construire pour instruire, 1519-2019, Lyon, Lieux Dits, coll. « Parcours du patrimoine », 2019.

12. POUPARD Laurent, "Paul Guadet et les écoles nationales d'horlogerie de Besançon et d'optique de Morez », In Situ. Revue des patrimoines, $\mathrm{n}^{\circ}$ 15, « Le patrimoine des guides : lectures de l'espace urbain européen ", 2011 [en ligne], http://journals.openedition.org/insitu/730 [lien valide en mars 2021].

13. POUPARD Laurent, "Ensemble flou et conservation au lycée. Le patrimoine mobilier des anciennes écoles nationales professionnelles d'horlogerie de Besançon et d'optique de Morez », In Situ. Revue des patrimoines, $\mathrm{n}^{\circ} 29$, «Ensembles mobiliers, industriels, techniques. Connaissance, protection, conservation, présentation au public», 2016, [en ligne] http:// journals.openedition.org/insitu/13019 [lien valide en mars 2021].

14. DUCROQUET-BLANCHET Sabrina, «La collaboration au cœur du processus de création. Les œuvres réalisées au titre du $1 \%$ artistique dans les lycées de Basse-Normandie (1951-1986)», In Situ. Revue des patrimoines, $\mathrm{n}^{\circ} 32$, «Le collectif à l'œuvre. Collaborations entre architectes et plasticiens ( $\mathrm{xx}^{\mathrm{e}}$ - $\mathrm{xxI}^{\mathrm{e}}$ siècles) », 2017 [en ligne] http://journals.openedition.org/insitu/15184 [lien valide en mars 2021].

15. Le colloque s'est tenu à Besançon et Morez du 9 au 11 avril 2013.

16. Les communications sont consultables en ligne : http://inventaire.aquitaine.fr/qui-sommesnous/journees-detude/les-patrimoines-des-lycees-en-nouvelle-aquitaine/ [lien valide en mars 2021]; certaines d'entre elles ont été publiées in CRON Éric (dir.), Arcades. Créations culturelles et patrimoines en Nouvelle-Aquitaine, numéro spécial, "Les Lycées, un patrimoine à découvrir », 2019, [en savoir plus via:] https://revue-arcades.fr/numero-special-patrimoinelycees/ [lien valide en mars 2021]

17. RENAUD-MORAND Bénédicte, Les Lycées dans la fabrique urbaine. Quelques cas de figure choisis en Région Auvergne-Rhône-Alpes avant inventaire (1802-1988), Lyon, Conseil régional AuvergneRhône-Alpes, 2020 [en ligne] https://hal.archives-ouvertes.fr/hal-02750967 [lien valide en mars 2021].

18. Requête de la Base Mérimée avril 2021. https://www.pop.culture.gouv.fr/search/list? base=\%5B\%22Patrimoine\%20architectural\%20\%28M\%C3\%A9rim\%C3\%A9e\%29\%22\%5D\&producteur=\%5B\%22Monuments\%20Historique 19. Requête de la Base Mérimée avril 2021 https://www.pop.culture.gouv.fr/search/list? base=\%5B \%22Patrimoine\%20architectural\%20\%28M\%C3\%A9rim\%C3\%A9e\%29\%22\%5D\&producteur=\%5B\%22Label\%20Architecture\%20c 
20. AMOUREUX Dominique et al., «Un art d'État ? Commandes publiques aux artistes plasticiens, 1945-1965 ", livret de visite dusite des Archives nationales de Pierrefitte-sur-Seine (31 mars-13 juillet 2017), Paris, Archives nationales, 2017, [disponible en ligne] https:// www.archives-nationales.culture.gouv.fr/documents/10157/124340/livret-expo-art-detat.pdf/ 9ee3b0ab-dae7-46db-b7cd-ca0b5e7b3c6e [lien valide en mars 2021]. La publication d'un ouvrage a accompagné l'exposition: HOTTIN Christian \& ROULLIER Clothilde (dir.), Un art d'État? Commandes publiques aux artistes plasticiens, 1945-1965, Rennes, Presses universitaires de Rennes / Archives nationales, 2017. 